\title{
RESULTS FROM X-RAY SURVEYS WITH ASCA
}

\author{
Yoshihiro Ueda \\ Institute of Space and Astronautical Science, Kanagawa 229-8510, Japan
}

\begin{abstract}
We present main results from X-ray surveys performed with $A S C A$, focusing on the $A S C A$ Large Sky Survey (LSS), the Lockman Hole deep survey, and the $A S C A$ Medium Sensitivity Survey (AMSS or the GIS catalog project). The $\log N$ - Log $S$ relations, spectral properties of sources, and results of optical identification are summarized. We discuss implications of these results for the origin of the $\mathrm{CXB}$.
\end{abstract}

KEYWORDS: diffuse radiation — surveys — galaxies: active — X-rays: galaxies

\section{INTRODUCTION}

Understanding the origin of the Cosmic X-ray Background (CXB or XRB) and cosmological evolution of X-ray extragalactic populations is one of the main goals of X-ray astronomy. In the soft X-ray band, the ROSAT satellite resolved $80 \%$ of the 0.5-2 keV CXB into individual sources (Hasinger et al. 1998) and optical identification revealed that the major population is type-I AGNs (Schmidt et al. 1998). Because of the technical difficulties, imaging sky surveys in the hard X-ray band (above $2 \mathrm{keV}$ ), where the bulk of the CXB energy arises, were not available until the launch of $A S C A$. The sensitivity limits achieved by previous mission such as HEAO1 (Piccinotti et al. 1982) and Ginga (Kondo et al. 1991) are at most $\sim 10^{-11} \mathrm{erg} \mathrm{s}^{-1} \mathrm{~cm}^{-2}(2-10 \mathrm{keV})$, and the sources observed by them only account for $3 \%$ of the CXB intensity in the $2-10 \mathrm{keV}$ band. In particular, there is a big puzzle on the CXB origin, called the "spectral paradox": bright AGNs observed with HEAO1, EXOSAT and Ginga have spectra with an average photon index of $\Gamma=1.7-1.9$ (e.g., Williams et al. 1992), which is significantly softer than that of the CXB itself $(\Gamma \simeq 1.4$; e.g., Gendreau et al. 1995). Furthermore, the broad band properties of sources at fluxes from $\sim 10^{-11}$ to $\sim 10^{-13} \mathrm{erg} \mathrm{s}^{-1} \mathrm{~cm}^{-2}(2-10 \mathrm{keV})$ are somewhat puzzling according to previous studies. The extragalactic source counts in the soft band (0.3-3.5 keV) obtained by Einstein Extended Medium Sensitivity Survey (EMSS; Gioia et al. 1990) is about 2-3 times smaller than that in the hard band (2-10 keV) obtained by the Ginga fluctuation analysis (Butcher et al. 1997) when we assume a power-law photon index of 1.7 .

The ASCA satellite (Tanaka, Inoue, \& Holt 1994), launched in 1993 February, was expected to change this situation. It is the first imaging satellite capable of 
TABLE 1 . Summary of $A S C A$ Surveys

\begin{tabular}{lll}
\hline \hline Survey Project & $\begin{array}{l}\text { Area } \\
\left(\mathrm{deg}^{2}\right)\end{array}$ & $\begin{array}{l}\text { Sensitivity }(2-10 \mathrm{keV}) \\
\left(\mathrm{erg} \mathrm{s}^{-1} \mathrm{~cm}^{-2}\right)\end{array}$ \\
\hline Large Sky Survey (LSS) & 7.0 & $1.5 \times 10^{-13}$ \\
Deep Sky Survey (DSS) & 0.3 & $4 \times 10^{-14}$ \\
Lockman Hole Deep Survey & 0.2 & $4 \times 10^{-14}$ \\
Survey of deep ROSAT fields & 1.0 & $5 \times 10^{-14}$ \\
$A S C A$ Medium-Sensitivity Survey (AMSS) & 110 & $7 \times 10^{-14}$ \\
\hline
\end{tabular}

study of the X-ray band above $2 \mathrm{keV}$ with a sensitivity up to several $10^{-14} \mathrm{erg} \mathrm{s}^{-1}$ $\mathrm{cm}^{-2}(2-10 \mathrm{keV})$ and covers the wide energy band from 0.5 to $10 \mathrm{keV}$, which allows us to directly compare results of the energy bands below and above $2 \mathrm{keV}$ with single detectors, hence accompanied with much less uncertainties than previous studies. By taking these advantages, several X-ray surveys have been performed with $A S C A$ to reveal the nature of hard X-ray populations: the ASCA Large Sky Survey (LSS; Ueda et al. 1998), the ASCA Deep Sky Survey (DSS; Ogasaka et al. 1998; Ishisaki et al. 1999 for the Lockman Hole), the ASCA Medium-Sensitivity Survey (AMSS or the GIS catalog project: Ueda et al. 1997, Takahashi et al. 1998, Ueda et al. 1999b; see also Cagnoni, Della Ceca, \& Maccacaro 1998 and Della Ceca et al. 1999), a survey of ROSAT deep fields (Georgantopoulos et al. 1997; Boyle et al. 1998), and so on. The sensitivity limits and survey area are summarized in Table 1 . In this paper, we present main results of the $A S C A$ surveys, focusing on the LSS ( $\S 2$ ), the Lockman Hole deep survey ( $(3)$, and the AMSS ( $\S 4)$. In $\S 5$, we summarize these results and discuss their implications for the origin of the CXB.

\section{THE LARGE SKY SURVEY}

\subsection{X-ray Data}

The survey field of the ASCA Large Sky Survey (LSS; Ueda et al., 1998) is a continuous region near the north Galactic pole, centered at $R A(2000)=13^{\mathrm{h}} 14^{\mathrm{m}}$, $\operatorname{DEC}(2000)=31^{\circ} 30^{\prime}$. Seventy-six pointings have been made over several periods from Dec. 1993 to Jul. 1995. The total sky area observed with the GIS and SIS amounts to $7.0 \mathrm{deg}^{2}$ and $5.4 \mathrm{deg}^{2}$ with the mean exposure time of $56 \mathrm{ksec}$ (sum of GIS2 and GIS3) and $23 \mathrm{ksec}$ (sum of SIS0 and SIS1), respectively. From independent surveys in the total $(0.7-7 \mathrm{keV})$, hard $(2-10 \mathrm{keV})$, and soft $(0.7-2 \mathrm{keV})$ bands, 107 sources are detected with sensitivity limits of $6 \times 10^{-14}, 1 \times 10^{-13}$, and $2 \times 10^{-14}$ $\operatorname{erg~s}^{-1} \mathrm{~cm}^{-2}$, respectively. The Log $N$ - Log $S$ relations derived from the LSS are summarized in Ueda et al. (1999a) together with a complete X-ray source list. At these flux limits, $30( \pm 3) \%$ of the CXB in the $0.7-7 \mathrm{keV}$ band and $23( \pm 3) \%$ in the 2-10 keV band have been resolved into discrete sources. The 2-10 keV Log $N-\log$ $S$ relation combined with the AMSS result (§4) is plotted in Figure 3. 
The spectral properties of the LSS sources suggest that contribution of sources with hard energy spectra become significant at a flux of $\sim 10^{-13} \mathrm{erg} \mathrm{s}^{-1} \mathrm{~cm}^{-2}(2-10$ $\mathrm{keV}$ ), which are different from the major population in the soft band. The average $2-10 \mathrm{keV}$ photon index is $1.49 \pm 0.10$ ( $1 \sigma$ statistical error in the mean value) for 36 sources detected in the $2-10 \mathrm{keV}$ band with fluxes below $4 \times 10^{-13} \mathrm{erg} \mathrm{s}^{-1} \mathrm{~cm}^{-2}$, whereas it is $1.85 \pm 0.22$ for 64 sources detected in the $0.7-2 \mathrm{keV}$ band with fluxes below $3 \times 10^{-13} \mathrm{erg} \mathrm{s}^{-1} \mathrm{~cm}^{-2}$. The average spectrum of 74 sources detected in the $0.7-7 \mathrm{keV}$ band with fluxes below $2 \times 10^{-13}$ shows a photon index of $1.63 \pm 0.07$ in the $0.7-10 \mathrm{keV}$ range: this index is consistent with the comparison of source counts between the hard and the soft band.

To investigate the X-ray spectra of these hard sources, we made deep follow-up observations with $A S C A$ for the five hardest sources in the LSS, selected by the apparent $0.7-10 \mathrm{keV}$ photon index from the source list excluding very faint sources. The results are summarized in Ueda et al. (1999c); see also Sakano et al. (1998) and Akiyama et al. (1998) for AX J131501+3141, the hardest source in the LSS. Three sources in this sample are optically identified as narrow-line AGNs and one is a weak broad-line AGN by Akiyama et al. (2000); one is not identified yet. We found that spectra of these sources are most likely subject to intrinsic absorption at the source redshift with column densities of $N_{\mathrm{H}}=10^{22} \sim 10^{23} \mathrm{~cm}^{-2}$.

\subsection{Optical Identification}

Akiyama et al. (2000) summarize the results of optical identification for a subsample of the LSS sources, consisting of 34 sources detected in the $2-7 \mathrm{keV}$ band with the SIS. The major advantage of this sample compared with other $A S C A$ surveys is good position accuracy; it is 0.6 arcmin in $90 \%$ radius from the $A S C A$ data alone, thanks to superior positional resolution of the SIS. To improve the position accuracy further, we made follow-up observations with ROSAT HRI over a part of the LSS field in Dec. 1997. Optical spectroscopic observations were made using the University of Hawaii $88^{\prime \prime}$ telescope, the Calar Alto 3.5m telescope, and the Kitt Peak National Observatories Mayall $4 \mathrm{~m}$ and $2.1 \mathrm{~m}$ telescopes.

Out of the 34 sources, 30 are identified as AGNs, 2 are clusters of galaxies, 1 is a Galactic star, and only 1 object remains unidentified. The identification as AGNs is based on existence of a broad emission line or the line ratios of narrow emission lines ([NII] $6583 \AA / \mathrm{H} \alpha$ and/or [OIII] $5007 \AA / \mathrm{H} \beta)$; see Akiyama et al. 2000 and references therein. Figure 1(a) shows the correlation between the redshift and the apparent photon index in the $0.7-10 \mathrm{keV}$ range, which is obtained from a spectral fit assuming no intrinsic absorption, for the identified objects. The 5 sources that have an apparent photon index smaller than 1.0 are identified as 4 narrow-line AGNs and 1 weak broad-line AGN, all are located at redshift smaller than 0.5. On the other hand, X-ray spectra of the other AGNs are consistent with those of nearby type 1 Seyfert galaxies. Four high redshift broad-line AGNs show somewhat apparently hard spectra with an apparent photon index of $1.3 \pm 0.3$, although it may be still marginal due to the limited statistics. 
To avoid complexity in classifying the AGNs by the optical spectra, we divide the identified AGNs into two using the X-ray data: the "absorbed" AGNs which show intrinsic absorption with a column density of $N_{\mathrm{H}}>10^{22} \mathrm{~cm}^{-2}$ and the "lessabsorbed" AGNs with $N_{\mathrm{H}}<10^{22}$. Correcting the flux sensitivity for different X-ray spectra, we found the contribution of the absorbed AGNs is almost comparable to that of less-absorbed AGNs in the $2-10 \mathrm{keV}$ source counts at a flux limit of $2 \times 10^{-13} \mathrm{erg} \mathrm{s}^{-1} \mathrm{~cm}^{-2}$. Figure 1(b) shows the correlation between the redshift and the $2-10 \mathrm{keV}$ luminosity of the identified AGNs. The redshift distribution of the 5 absorbed AGNs is concentrated at $z<0.5$, which contrasts to the presence of 15 less-absorbed AGNs at $z>0.5$. This suggests a deficiency of AGNs with column densities of $N_{\mathrm{H}}=10^{22-23}$ at $z=0.5-2$, or in the X-ray luminosity range larger than $10^{44} \mathrm{erg} \mathrm{s}^{-1}$, or both. Note that if the 4 broad-line AGNs with hard spectra have intrinsic absorption instead of other hardening mechanism such as Compton reflection, it could complement this deficiency.
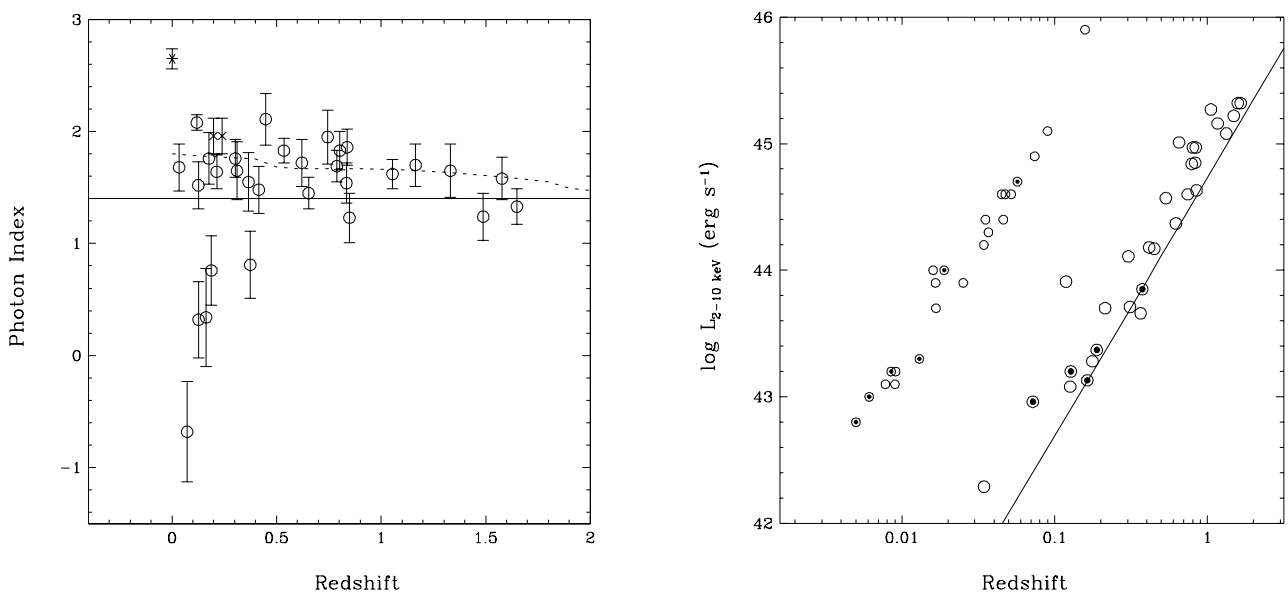

FIGURE 1. (a) left: the correlation between the redshift and the apparent 0.7-10 $\mathrm{keV}$ photon index for the identified objects in the LSS (Akiyama et al. 2000). The open circles, crosses, and asterisk represent AGNs, clusters of galaxies, and a Galactic star, respectively. The dotted curve shows the expected apparent photon index in the observed $0.7-10 \mathrm{keV}$ band as a function of redshift, for a typical spectrum of type-1 Seyfert galaxies with a Compton reflection component. (b) right: The 2-10 $\mathrm{keV}$ luminosity versus redshift diagram for the LSS AGNs (with large open circles, Akiyama et al. 2000), and for the HEAO1A2 AGNs (with small marks, Piccinotti et al. 1982). The "absorbed" AGNs are plotted with dots. Lines indicate detection limits of the LSS for a source with an photon index of 1.7 with no intrinsic absorption. 


\section{THE LOCKMAN HOLE DEEP SURVEY}

Deep surveys were performed with $A S C A$ over several fields (Ogasaka et al. 1998), although optical identification is more difficult than the LSS because of faint flux levels and source confusion problem. To overcome this difficulty, we have been conducting a deep survey of the Lockman Hole, where the ROSAT deep survey was performed (Hasinger et al. 1998). The advantage of selecting this field is that we already have a complete soft X-ray source catalog down to a flux limit of $5.5 \times 10^{-15}$ erg s${ }^{-1} \mathrm{~cm}^{-2}(0.5-2 \mathrm{keV})$, most of which have been optically identified (Schmidt et al. 1998). In addition, we utilized an X-ray source list at even fainter flux limits (G. Hasinger, private communication). Since the flux limits of the ROSAT surveys are extremely low, we can expect most of $A S C A$ sources could have ROSAT counterparts within reasonable range of spectral hardness. Utilizing positions of the ROSAT sources, we can determine the hard-band flux for individual sources, which would otherwise have been difficult to separate, to a flux limit of $3 \times 10^{-14} \mathrm{erg} \mathrm{s}^{-1}$ $\mathrm{cm}^{-2}(2-10 \mathrm{keV})$. Preliminary results are reported in Ishisaki et al. (1999).

Up to present, we have made 3 pointings in the direction of the Lockman Hole with $A S C A$ on 1993 May 22-23, 1997 April 29-30, and 1998 November 27 for a net exposure of $63 \mathrm{ksec}$ (average of the 8 SIS chips), $64 \mathrm{ksec}$, and $62 \mathrm{ksec}$, respectively. The pointing positions were arranged so that the superposed image of the SIS field of views (FOVs) covers the PSPC and HRI FOVs as much as possible. We here used only the SIS data considering its superior positional resolution. Analysis was made through the 2-dimensional maximum-likelihood fitting to a raw, superposed image in photon counts space in the sky coordinates, with a model consisting of source peaks (point spread functions) and the background. As a first step, we put sources into the model at the positions of the ROSAT catalogs. Then, after checking the residual image of the fit, we added remaining peaks that were missing in the ROSAT catalogs. Thus, we determined the significance and flux of each source in three energy bands, $0.7-7 \mathrm{keV}, 2-7 \mathrm{keV}$, and $0.7-2 \mathrm{keV}$, including new sources detected with $A S C A$. We corrected for the degradation of detection efficiency caused by the radiation damage using the CXB intensity. Note that the $A S C A$ sensitivity limits strongly depends on position due to the multiple pointings and the vignetting of the XRT.

We detected 27 sources altogether with significances higher than $3.5 \sigma$ in either of the three survey bands. Two sources were newly detected with $A S C A$. One object is a variable source having a $0.7-7 \mathrm{keV}$ photon index of about 1.7 , which was very faint during the ROSAT observations. The other shows a very hard spectrum and is detected only in the $2-7 \mathrm{keV}$ band. In the combined SIS FOVs, 43 sources out of 50 sources in the Schmidt et al. (1998) catalog are located. Identification of $A S C A$ sources using the ROSAT catalog is summarized in Table 2. Since the number of sources detected in the $2-7 \mathrm{keV}$ band is limited due to poor photon statistics, we here use the results for 25 sources detected in the $0.7-7 \mathrm{keV}$ band for comparison with the ROSAT survey. Four unidentified sources in the $A S C A$ survey have ROSAT counterparts in the deeper X-ray source catalog (G. Hasinger, private 
TABLE 2. Summary of optical identification of the $A S C A$ Lockman Hole deep survey by the ROSAT catalog (Schmidt et al. 1998)

\begin{tabular}{lcc}
\hline \hline Population & ROSAT & ASCA $(0.7-7 \mathrm{keV})$ \\
\hline Total & 43 & 25 \\
\hline Type-1 AGN (a-c) & 26 & 13 \\
Type-2 AGN (d-e) & 7 & 6 \\
Group/Galaxies & 3 & 0 \\
Star & 3 & 1 \\
Unidentified & 4 & $4+1$ \\
\hline
\end{tabular}

communication) and remaining one is the variable source detected only with $A S C A$. For AGNs identified by Schmidt et al. (1998), we divided them into two according to their optical spectra: (1) type-1 AGNs, corresponding to either of class a, b, or c, showing broad emission lines, and (2) type-2 AGN, class d or e, showing only narrow emission lines. As noticed from the table, 6 out of 7 type-2 AGNs were detected, whereas only half of the 26 type-1 AGNs were detected with $A S C A$, which covers much harder band than the ROSAT. This suggests that contribution of type2 AGNs are more dominant in higher energy bands than in the soft band at similar flux levels.

\section{THE ASCA MEDIUM-SENSITIVITY SURVEY}

Because these surveys are limited in sky coverage, the sample size is not sufficient to obtain a self-consistent picture about the evolution of the sources over the wide fluxes, from $\sim 10^{-11} \mathrm{erg} \mathrm{s}^{-1} \mathrm{~cm}^{-2}(2-10 \mathrm{keV})$ which is the sensitivity limit of HEAO1 A2 (Piccinotti et al. 1982), down to $\sim 10^{-13} \mathrm{erg} \mathrm{s}^{-1} \mathrm{~cm}^{-2}(2-10 \mathrm{keV}$ ), that of $A S C A$. To complement these shortcomings, we have been working on the project called the "ASCA Medium Sensitivity Survey (AMSS)", or the GIS catalog project. In the project, we utilize the GIS data from the fields that have become publicly available to search for serendipitous sources. The large field of view and the low-background characteristics make the GIS instrument ideal for this purpose.

Main results from the AMSS are reported in Ueda et al. (1999b), which were obtained from selected GIS fields of $|b|>20^{\circ}$ observed from 1993 to 1996, covering the total sky area of $106 \mathrm{deg}^{-2}$. The sample contains 714 serendipitous sources, of which 696,323 , and 438 sources are detected in the $0.7-7 \mathrm{keV}$ (total), $2-10 \mathrm{keV}$ (hard), and $0.7-2 \mathrm{keV}$ (soft) band, respectively. This is currently the largest X-ray sample covering the $0.7-10 \mathrm{keV}$ band. Figure 2 (a) shows the correlation between the 0.7-7 keV flux and the hardness ratio between the $2-10 \mathrm{keV}$ and $0.7-2 \mathrm{keV}$ count rates. We also plot the average hardness ratio in several flux ranges, separated by the dashed curves, with crosses. It is clearly seen that the average spectrum becomes harder with a decreasing flux: the corresponding photon index (assuming 
The Log N - Log S Relation $(0.7-7 \mathrm{keV})$
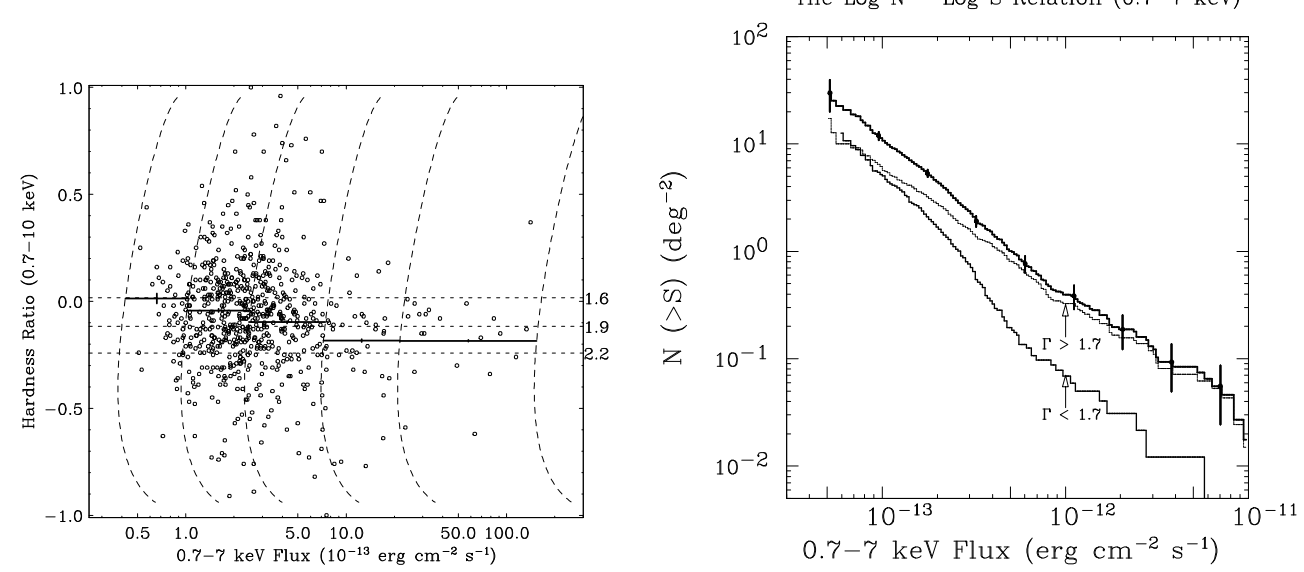

FIGURE 2. (a) left: The correlation between the $0.7-7 \mathrm{keV}$ flux and the hardness ratio between the $0.7-2 \mathrm{keV}$ and $2-10 \mathrm{keV}$ count rates for sources detected in the $0.7-$ $7 \mathrm{keV}$ survey in the AMSS sample (Ueda et al. 1999b). The crosses show the average hardness ratios (with $1 \sigma$ errors in the mean value) in the flux bin separated by the the dashed curves, at which the count rate hence the sensitivity limit is constant. The dotted lines represent the hardness ratios corresponding to a photon index of 1.6, 1.9, and 2.2 assuming a power law spectrum. (b) right: The integral Log $N$ $\log S$ relations in the $0.7-7 \mathrm{keV}$ survey band, derived from the AMSS sample. The medium-thickness curve represents the result for the hard source sample, consisting of sources with an apparent $0.7-10 \mathrm{keV}$ photon index $\Gamma$ smaller than 1.7, the thin curve represents that for the soft source sample (with $\Gamma$ larger than 1.7), and the thick curve represents the sum. The $90 \%$ statistical errors in source counts are indicated by horizontal bars at several data points.

a power law over the $0.7-10 \mathrm{keV}$ band with no absorption) changes from 2.1 at the flux of $\sim 10^{-11} \mathrm{erg} \mathrm{s}^{-1} \mathrm{~cm}^{-2}$ to 1.6 at $\sim 10^{-13} \mathrm{erg} \mathrm{s}^{-1} \mathrm{~cm}^{-2}(0.7-7 \mathrm{keV})$. Similar hardening are also reported in the $2-10 \mathrm{keV}$ range by Della Ceca et al. (1999) using 60 serendipitous sources. Figure 2(b) shows the integral $\log N-\log$ $S$ relations in the $0.7-7 \mathrm{keV}$ survey band for the soft source sample, consisting of sources with an apparent $0.7-10 \mathrm{keV}$ photon index larger than 1.7, and for the hard source sample, with an index smaller than 1.7. This demonstrates that sources with hard energy spectra in the $0.7-10 \mathrm{keV}$ range are rapidly increasing with decreasing fluxes, compared with softer sources.

\section{SUMMARY}

The $A S C A$ surveys have brought a clear, self-consistent picture about statistical properties of sources that constitute about $30 \%$ of the CXB in the broad energy 
band of $0.7-10 \mathrm{keV}$. Figure 3 summarizes the $2-10 \mathrm{keV} \log N-\log S$ relation obtained from the $A S C A$ surveys together with the results from previous missions. The direct source counts from combined results of the LSS (Ueda et al. 1999b) and the AMSS (Ueda et al. 1999c; these contain the data used by Cagnoni, Della Ceca, \& Maccacaro 1998) give the tightest constraints so far over a wide flux range from $\sim 10^{-11}$ to $\sim 7 \times 10^{-14} \mathrm{erg} \mathrm{s}^{-1} \mathrm{~cm}^{-2}: N(>S)=16.8 \pm 7.2$ (90\% statistical error), $11.43 \pm 2.4,3.76 \pm 0.42,1.08 \pm 0.17$, and $0.33 \pm 0.09 \mathrm{deg}^{-2}$, at $S=7.4 \times 10^{-14}, 1.0 \times$ $10^{-13}, 2.0 \times 10^{-13}, 4.0 \times 10^{-13}$, and $1.0 \times 10^{-12} \mathrm{erg} \mathrm{s}^{-1} \mathrm{~cm}^{-2}$, respectively. The DSS gives a direct source counts at the faintest flux, $3.8 \times 10^{-14} \mathrm{erg} \mathrm{s}^{-1} \mathrm{~cm}^{-2}$ (Ogasaka et al. 1998), whereas the fluctuation analysis of deep SIS fields constrains the Log $N$ - $\log S$ relation at fluxes down to $1.5 \times 10^{-14}$ (Gendreau, Barcons, \& Fabian 1998). As seen from the figure, the $A S C A$ direct source counts smoothly connect the two regions constrained by the Ginga and $A S C A$ fluctuation analysis.

The AMSS/LSS results demonstrate that the average spectrum of X-ray sources becomes harder toward fainter fluxes: the apparent photon index in the $0.7-10 \mathrm{keV}$ range changes from 2.1 at the flux of $\sim 10^{-11}$ to 1.6 at $\sim 10^{-13} \mathrm{erg} \mathrm{s}^{-1} \mathrm{~cm}^{-2}(2-10$ $\mathrm{keV})$. This fact can be explained by the rapid emergence of population with hard energy spectra, as is clearly indicated in Figure 2(b). The evolution of broad-band properties of sources solves the puzzle of discrepancy discrepancy of the source counts between the soft (EMSS) and the hard band (Ginga and HEAO1). If we compare the $A S C A \log N$ - $\log S$ relations (including Galactic objects) between above and below $2 \mathrm{keV}$, the hard band source counts at $S \sim 10^{-13} \mathrm{erg} \mathrm{s}^{-1} \mathrm{~cm}^{-2}$ $(2-10 \mathrm{keV})$ matches the soft band one when we assume a photon index of 1.6 for flux conversion, whereas at brighter level of $S=4 \times 10^{-13} \sim 10^{-12} \mathrm{erg} \mathrm{s}^{-1} \mathrm{~cm}^{-2}(2-10$ $\mathrm{keV}$ ), we have to use a photon index of about 1.9 to make them match. The latter fact is consistent with the average $0.7-10 \mathrm{keV}$ spectrum at the same flux levels, and can be connected the the "soft" spectrum of the fluctuation observed with Ginga, which shows a photon index of $1.8 \pm 0.1$ in the $2-10 \mathrm{keV}$ range (Butcher et al. 1997).

The optical identification revealed that the major population at fluxes of $10^{-13}$ $\mathrm{erg} \mathrm{s}^{-1} \mathrm{~cm}^{-2}$ are AGNs. The population of hard sources, which are most responsible for making the average spectrum hard, are X-ray absorbed sources. They are mostly identified as narrow line (type-2) AGNs. The contribution of these type-2 AGNs is larger in the hard band than in the soft band at the same flux limit. Recent results of the 5-10 keV band survey by BeppoSAX confirms this tendency (Fiore et al. 1999). These results support the scenario that the CXB consists of unabsorbed AGNs and absorbed AGNs, whose contribution becomes more significant with decreasing fluxes and in harder energy band.

We found, however, possible evidence that is not consistent with the "unified scheme" of AGNs (e.g., Awaki et al. 1991), on which many AGN synthesis models are based. The LSS results may imply deficiency of X-ray luminous, absorbed AGNs, with $N_{\mathrm{H}}=10^{22-23}$ at $z=0.5-2$, or in the X-ray luminosity range larger than $10^{44}$ $\mathrm{erg} \mathrm{s}^{-1}$, although we cannot rule out possibility, for example, that there are many luminous AGNs at $z>2$ with extreme heavy absorption of $N_{\mathrm{H}}>10^{24}$. On the other hand, there is another implication that there could be a population of AGNs 
at high redshifts $(z>1)$ that are optically identified as type-1 AGNs but have apparently hard X-ray spectra, although the origin of the hardness is not clear yet. Future surveys by Chandra and XMM together with optical identification of the AMSS sources will reveal the luminosity, number, and spectral evolutions of extragalactic populations including absorbed AGNs, which will eventually lead us to full understanding of the origin of the CXB.

\section{ACKNOWLEDGEMENTS}

I thank all the collaborators of our $A S C A$ survey projects, especially, M. Akiyama, G. Hasinger, H. Inoue, Y. Ishisaki, I. Lehmann, K. Makishima, Y. Ogasaka, T. Ohashi, K. Ohta, M. Sakano, T. Takahashi, T. Tsuru, W. Voges, T. Yamada, and A. Yamashita.

\section{REFERENCES}

Akiyama, M., et al. 1998, ApJ, 500, 173

Akiyama, M., et al. 2000, ApJ, in press

Awaki, H., Koyama, K., Inoue, H., \& Halpern, J.P. 1991, PASJ, 43, 195

Boyle, B.J., et al. 1998, MNRAS, 296, 1

Butcher, J.A., et al. 1997, MNRAS, 291, 437

Cagnoni, I., Della Ceca, R., \& Maccacaro, T. 1998, ApJ, 493, 54

Della Ceca, R., et al. 1999, ApJ, 524, 674

Fiore, F., et al. 1999, MNRAS, 306, L55

Gendreau, K.C., et al. 1995, PASJ, 47, L5

Gendreau, K.C., Barcons, X., \& Fabian, A.C. 1998, MNRAS, 297, 41

Georgantopoulos, I., et al. 1997, MNRAS, 291, 203

Gioia, I.M. et al. 1990, ApJS, 72, 567

Hasinger, G. 1998, AN, 319, 37

Hasinger, G., et al. 1998, A\&A, 329, 482

Ishisaki, Y., et al. 1999, Advanced in Space Research, in press

Kondo, H. et al. 1982, in "Frontiers of X-ray Astronomy", Universal Academy Press, Tokyo, p. 655

Ogasaka, Y., et al. 1998, Astro. Nachr., 319, 43

Piccinotti, G., et al. 1982, ApJ, 253, 485

Sakano, M. et al. 1998, ApJ, 505, 129

Schmidt, M., et al. 1998, A\&A, 329, 495

Takahashi, T., Ueda, Y., Ishisaki, Y., Ohashi, T., \& Makishima, K. 1998, AN, 319, 91

Tanaka, Y., Inoue, H., \& Holt, S.S. 1994, PASJ, 46, L37

Ueda, Y., Takahashi, T., Ishisaki, Y., Makishima, K. \& 1997, in "All-Sky X-Ray Observations in the Next Decade", RIKEN, Saitama, p55

Ueda, Y., et al. 1998, Nature, 391, 866

Ueda, Y., et al. 1999a, ApJ, 518, 656

Ueda, Y., Takahashi, T., Ishisaki, Y., Ohashi, T., \& Makishima, K. 1999b, ApJ, 524, L11

Ueda, Y., et al. 1999c, Advanced in Space Research, in press

Williams, O.R. et al. 1992, ApJ, 389, 157 


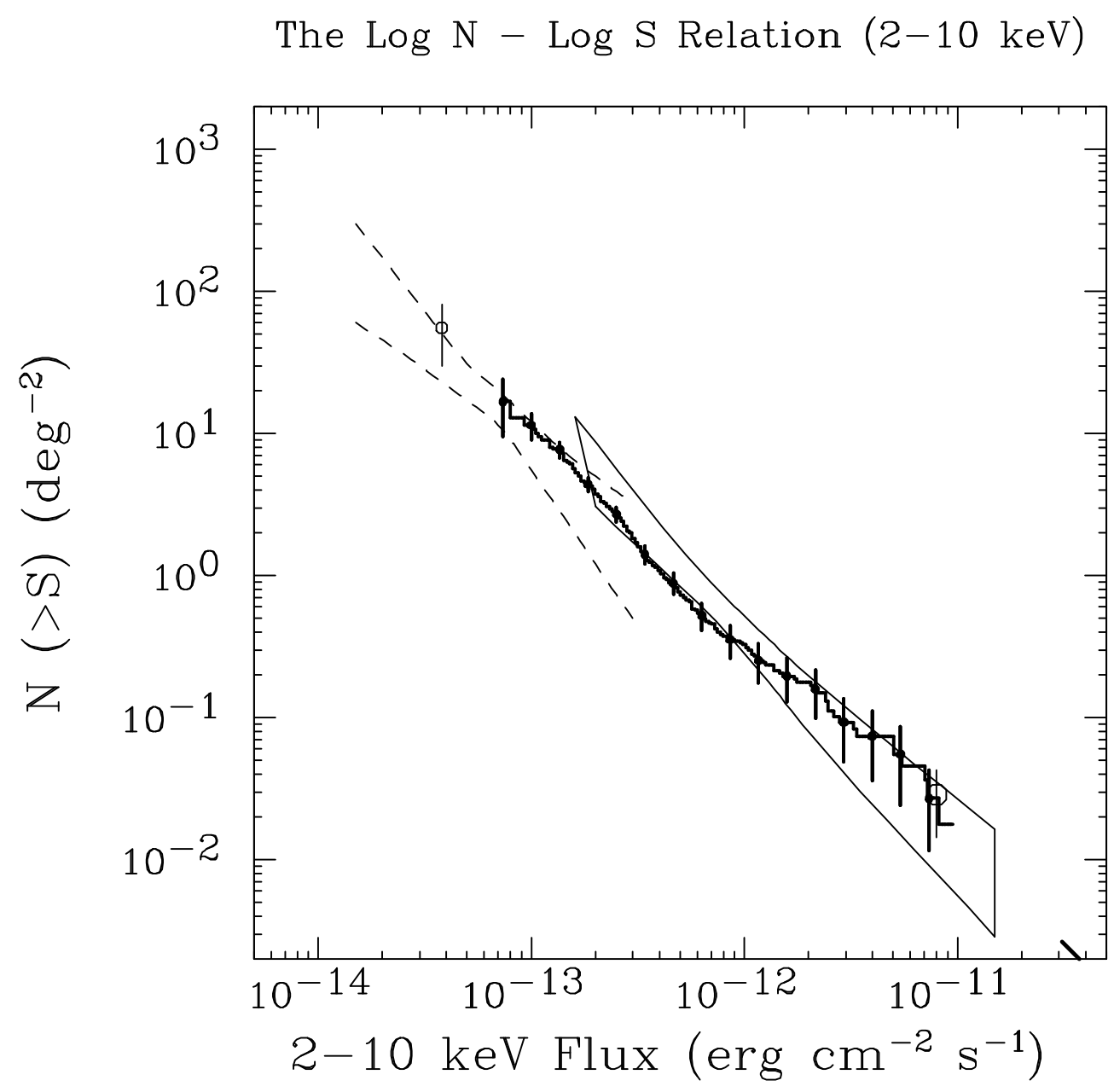

FIGURE 3. Summary of the $2-10 \mathrm{keV} \log N-\log S$ relation obtained by the $A S C A$ surveys, compared with previous results. The steps are the combined results from the LSS (Ueda et al. 1998) and the AMSS (Ueda et al. 1999b). The faintest point at $4 \times 10^{-14} \mathrm{erg} \mathrm{s}^{-1} \mathrm{~cm}^{-2}$ is derived from the DSS utilizing the SIS data (Ogasaka et al. 1998). The trumpet shape between two dashed lines indicates $1 \sigma$ error region from the fluctuation analysis of ASCA SIS deep fields (Gendreau, Barcons \& Fabian 1998). The contour at $10^{-13} \sim 10^{-11} \mathrm{erg} \mathrm{s}^{-1} \mathrm{~cm}^{-2}$ represents the constraints by the Ginga fluctuation analysis at 90\% confidence level (Butcher et al. 1997). The open circle at $8 \times 10^{-12} \mathrm{erg} \mathrm{s}^{-1} \mathrm{~cm}^{-2}$ corresponds to the source count by Ginga survey (Kondo et al. 1991), and the thick-line above $3 \times 10^{-11} \mathrm{erg} \mathrm{s}^{-1} \mathrm{~cm}^{-2}$ is the extragalactic $\log N-\log S$ relation determined by HEAO1 A2 (Piccinotti et al. 1982). All the horizontal bars represent $90 \%$ statistical errors in source counts. 